\title{
Study of 3D-growth conditions for selective area MOVPE of high aspect ratio GaN fins with non-polar vertical sidewalls
}

\author{
Jana Hartmann $^{a, b^{*}}$, Frederik Steib $^{a, b}$, Hao Zhou $^{a, b}$, Johannes Ledig $^{a, b, e}$, Lars Nicolai $^{d}$, Sönke Fündling ${ }^{a, b}$, Tilman \\ Schimpke ${ }^{c}$, Adrian Avramescuc, Tansen Varghese ${ }^{c}$, Achim Trampert ${ }^{d}$, Martin Straßburg ${ }^{c}$, Hans-Jürgen \\ Lugauer $^{b, c}$, Hergo-Heinrich Wehmann ${ }^{a, b}$, Andreas Waag ${ }^{a, b}$ \\ ${ }^{a}$ Institut für Halbleitertechnik and Laboratory for Emerging Nanometrology, Technische Universität \\ Braunschweig, 38092 Braunschweig, Germany \\ bepitaxy competence center ec ${ }^{2}$, Hans-Sommer-Straße 66, 38106 Braunschweig, Germany \\ 'OSRAM Opto Semiconductors GmbH, Leibnizstraße 4, 93055 Regensburg, Germany \\ dPaul-Drude-Institut für Festkörperelektronik, Hausvogteiplatz 5-7, 10117 Berlin, Germany \\ ephysikalisch-Technische Bundesanstalt (PTB), Bundesallee 100, 38116 Braunschweig, Germany \\ *corresponding author: Jana.Hartmann@tu-bs.de
}

\begin{abstract}
$\mathrm{GaN}$ fins are 3D architectures elongated in one direction parallel to the substrate surface. They have the geometry of walls with a large height to width ratio as well as small footprints. When appropriate symmetry directions of the GaN buffer are used, the sidewalls are formed by non-polar $\{11-20\}$ planes, making the fins particularly suitable for many device applications like LEDs, FETs, lasers, sensors or waveguides. The influence of growth parameters like temperature, pressure, V/III ratio and total precursor flow on the fin structures is analyzed. Based on these results, a 2-temperature-step-growth was developed, leading to fins with smooth side and top facets, fast vertical growth rates and good homogeneity along their length as well as over different mask patterns. For the core-shell growth of fin LED heterostructures, the 2temperature-step-growth shows much smoother sidewalls and less crystal defects in the InGaN QW and p$\mathrm{GaN}$ shell compared to structures with cores grown in just one step. Electroluminescence spectra of the 2temperature-step-grown fin LED are demonstrated.
\end{abstract}

Keywords: A1. Crystal morphology, A3. Metalorganic vapor phase epitaxy, B.1 Gallium compounds, B.1 Nitrides, B.3 Light emitting diodes

\section{Introduction}

The material system of III-nitrides is interesting for many electronic, optoelectronic or sensor applications. $\mathrm{GaN}$ is the basis for high efficiency white light-emitting diodes (LEDs), high-electron-mobility transistors (HEMTs) and low energy-consumption gas sensors. The aim of improving these devices or endowing them with new features led to the development of three-dimensional (3D) GaN structures with high surface-tovolume ratios, semi- and non-polar surfaces and high crystal quality. The most famous representatives of these 3D structures are GaN columns, which are elongated in just one dimension perpendicular to the substrate surface. However, there are also other 3D architectures like fin geometries. These fins are also called stripes[1], sheets[2] or walls[3] and can be produced by top-down[4],[5] or bottom-up[6] approaches by molecular beam epitaxy (MBE) or metal organic vapor phase epitaxy (MOVPE). Especially for bottomup fabricated fins, there are many advantages. Compared to GaN planar structures, fins are assumed to have a lower dislocation density due to their high aspect ratio (AR), which can lead to annihilation of extended defects. Additionally, when grown along certain symmetry directions, fins have non-polar surface planes without spontaneous or piezoelectric polarization fields. Compared to vertical GaN columns and corresponding core-shell devices, fins and fin LEDs have several potential benefits[7]: higher mechanical stability, large-scale non-polar surfaces with less edge effects (when compared to the six edges on the hexagonal base of columns), higher active area ratios (as long as the pitch is larger than 1.7 times the width), less current density and heating in the footprint area (when the same current density through the active area is taken), a more reproducible MOVPE process for fins compared to columns and last but not least the possibility of a simplified characterization due to fast accessibility of fin cross-sections. All these properties of fins have been discussed in detail elsewhere[7]. 
In this publication, the applied continuous flux method for selective area growth (SAG) by MOVPE is described in detail. Especially the dependence of the fin structure on important parameters for 3D growth, i.e. temperature, pressure, V/III ratio and total precursor flows, is discussed in detail. Based on these results, a 2-temperature-step-growth is developed in order to achieve high AR fins with smooth top surfaces and with high homogeneity along their length for different mask patterns. Spatially resolved cathodoluminescence (CL) measurements and transmission electron microscopy (TEM) of fin cores as well as fin LEDs are presented.

\section{Materials and Methods}

$\mathrm{GaN}$ fins were grown by $\mathrm{SAG}$ on $\mathrm{SiO}_{x}$ passivated $\mathrm{GaN}$ on sapphire templates of 2" diameter. The $30 \mathrm{~nm}$ thick $\mathrm{SiO}_{\mathrm{x}}$ mask was prepared by chemical vapor deposition and was patterned by photolithography and reactive ion etching by inductively coupled plasma (ICP-RIE). The mask comprehend line openings in 12 different patterns (see Figure $1 \mathrm{~b} / \mathrm{c}$ ) with widths $\mathrm{W}_{\mathrm{p}}$ (width of pattern) of $0.5 \mu \mathrm{m}, 1 \mu \mathrm{m}, 1.5 \mu \mathrm{m}$ and $2 \mu \mathrm{m}$ as well as pitch-to-width ratios of 3, 6 and 9, respectively[7]. Directly after the ICP-RIE process, an $\mathrm{O}_{2}$ plasma treatment was applied for $120 \mathrm{~s}$ in order to remove all photoresist residuals from the template.

During SAG by MOVPE in a 3x2" Thomas Swan close coupled showerhead reactor, the templates were heated to $1060^{\circ} \mathrm{C}$ under $\mathrm{N}_{2}$ and ammonia $\left(\mathrm{NH}_{3}\right)$. Subsequently, the carrier gas was switched to $\mathrm{H}_{2}$ and a thin $\mathrm{GaN}$ layer grew in the mask openings during a $50 \mathrm{~s}$ long filling-step with trimethylgallium (TMGa) and $\mathrm{NH}_{3}$ under a high $\mathrm{V} / \mathrm{III}$ ratio of 1000 . Thereafter, the 3D-growth-step was performed under a low V/III ratio of 77, at a temperature of $1060{ }^{\circ} \mathrm{C}$ and a reactor pressure of $250 \mathrm{hPa}$. Total precursor flows were as high as $134 \mu \mathrm{mol} / \mathrm{min} \mathrm{TMGa}$ and $10.3 \mathrm{mmol} / \mathrm{min} \mathrm{NH}_{3}$. The growth time was $1800 \mathrm{~s}$ if not mentioned otherwise. These parameters were used as the standard 3D-growth-conditions, while several variations of these conditions will be listed in the following.

Samples with different temperatures during filling- and 3D-growth-step (varying from $1000{ }^{\circ} \mathrm{C}$ to $1100{ }^{\circ} \mathrm{C}$ with a step size of $20^{\circ}$ ) were grown. At a constant temperature of $1060^{\circ} \mathrm{C}$ the pressure during the 3Dgrowth-step was changed to $150 \mathrm{hPa}$ and $350 \mathrm{hPa}$, respectively. For the comparison of different $\mathrm{V} / \mathrm{III}$ ratios, samples with varying TMGa flow were grown at $1060^{\circ} \mathrm{C}$ : TMGa flows from 89 to $179 \mu \mathrm{mol} / \mathrm{min}$ (with constant $\mathrm{NH}_{3}$ flow of $10 \mathrm{mmol} / \mathrm{min}$ ) were used, leading to $\mathrm{V} / \mathrm{III}$ ratios of 116 to 58 . In another set of samples, the $\mathrm{NH}_{3}$ flow was varied (with a constant TMGa flow of $134 \mu \mathrm{mol} / \mathrm{min}$ ) from approx. 4 $\mathrm{mmol} / \mathrm{min}$ to approx. $17 \mathrm{mmol} / \mathrm{min}$, leading to $\mathrm{V} / \mathrm{III}$ ratios of 30 to 130 . While keeping the V/III ratio constant at 77, total flows from low $\left(67 \mu \mathrm{mol} / \mathrm{min} \mathrm{TMGa}, 5.2 \mathrm{mmol} / \mathrm{min} \mathrm{NH}_{3}\right)$ to high $(446 \mu \mathrm{mol} / \mathrm{min}$ TMGa, $34.4 \mathrm{mmol} / \mathrm{min} \mathrm{NH}$ ) were examined. Since none of the available $\mathrm{NH}_{3}$ mass flow controllers (MFCs) can regulate the flow over the whole range, two different MFCs were used subsequently for this series.

For the fins evolving during the 3D-growth-step always some overgrowth over the $\mathrm{SiO}_{\mathrm{x}}$ mask is visible, therefore the real width $\mathrm{W}_{\mathrm{r}}$ is generally larger than $\mathrm{W}_{\mathrm{p}}$. The width $\mathrm{W}_{\mathrm{r}}$ and height of the fins was measured by a Tescan Mira3 GMH field emission scanning electron microscope (FE-SEM) using the secondary electron (SE) signal. Each data point presented in this publication is the mean value of three to six readings measured at different points inside the corresponding pattern.

For epitaxy of fin LEDs, a shell was grown around the fin cores. This shell consists of an unintentionally doped GaN spacer, a single InGaN QW, another unintentionally doped GaN spacer and several p-GaN capping layers (from inside to outside). The QW was grown at $740{ }^{\circ} \mathrm{C}$ and $40 \mathrm{hPa}$. For activation of the p$\mathrm{GaN}$, the fin LEDs were annealed in the MOVPE reactor at $770{ }^{\circ} \mathrm{C}$ for $20 \mathrm{~min}$.

Electrical contacting inside the FE-SEM was performed by a Kleindiek MM3A-EM manipulator equipped with a tungsten probe tip. Imaging of the electron beam induced currents (EBIC) between two contacts is obtained by an integrated EBIC detector. Beside detectors for electron signals the FE-SEM is equipped with a Gatan MonoCL4 cathodoluminescence setup with a parabolic collection mirror. The CL and EBIC imaging were performed using an electron probe of $5 \mathrm{keV}$ and about $550 \mathrm{pA}$.

For microstructural investigations a JEOL JEM-3010 and JEM-2100F TEM were employed. The JEM$2100 \mathrm{~F}$ microscope is equipped with a scanning unit including a bright-field as well as a high-angle annular 
dark-field (HAADF) detector, which is mainly sensitive to the chemical composition (Z-contrast) of the sample. For the site-specific sample preparation two different preparation techniques were applied. For the first method, cross-sectional samples were conventionally prepared by embedding the fins in Gatan G1 epoxy and sandwiching the sample between sapphire dummies. Fine slices were mechanically grinded and dimpled and finally sputtered with argon. For the second method, lamellae were cut out of the wafer by using the $\mathrm{Ga}^{+}$focused ion beam in-situ lift-out (FIB-INLO) technique[8]. In order to protect the surface, the fin structures were embedded by Gatan G1-epoxy, which was diluted with acetone to reduce the viscosity during its application.

\section{Results}

In the discussion below, we are concentrating on fins with longitudinal expansions in the m-direction of the GaN buffer layer. In this case, their longer sidewalls are $\{11-20\}$ a-planes and hence are non-polar. Other directions turn out to show more semipolar and less non-polar facets[7]. Under standard growth conditions, as described in the section "Materials and Methods", the fins have increasing homogeneity with larger $\mathrm{W}_{\mathrm{p}}$ [7]. The smaller $\mathrm{W}_{\mathrm{p}}$, the more interruptions along the fin length emerge. For the smallest width $\mathrm{W}_{\mathrm{p}}=0.5$ $\mu \mathrm{m}$ almost no continuous fins are evolving. For widths of 1.5 and $2 \mu \mathrm{m}$, more homogenous fins arise. They have relatively smooth sidewall surfaces with just a few pits and steps, but rough top surfaces with a bubble-like morphology. After a growth time of $1800 \mathrm{~s}$, the fins in pattern W2 P12 (i.e. $\mathrm{W}_{\mathrm{p}}=2 \mu \mathrm{m}$, pitch $=$ $12 \mu \mathrm{m}$ ) have an average height and width of $10.4 \mu \mathrm{m}$ and $3.4 \mu \mathrm{m}$, respectively.
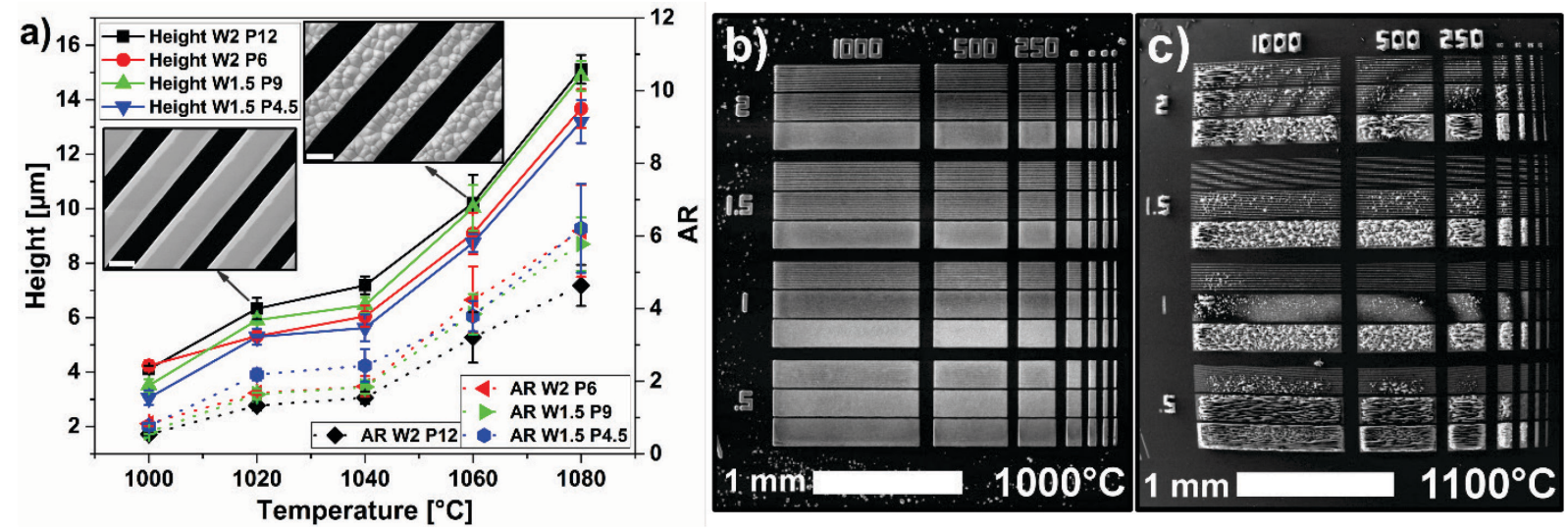

Figure 1: (a) Fin height and AR, given for different mask patterns (e.g. W2 P18 stands for the pattern with $\mathrm{W}_{\mathrm{p}}=2 \mu \mathrm{m}$ and pitch $=18 \mu \mathrm{m}$ ), is plotted versus growth temperature. The insets show top view SE images of fins grown in pattern $\mathrm{W} 1.5 \mathrm{P} 4.5$ at $1020^{\circ} \mathrm{C}$ and $1060^{\circ} \mathrm{C}$, respectively. The scale bar has a length of 2 $\mu \mathrm{m}$ in both cases. (b) and (c) Top view SE images of one field with all 12 patterns grown at $1000{ }^{\circ} \mathrm{C}$ and $1100{ }^{\circ} \mathrm{C}$, respectively.

\subsection{Temperature}

The temperature during SAG has a large influence on the structure of the fins as well as on their homogeneity. Whereas the width $\left(\mathrm{W}_{\mathrm{r}}\right)$ is decreasing with increasing temperature, the height of the fins is increasing distinctly. The latter is enhanced by up to a factor of 4 with a temperature shift from $1000{ }^{\circ} \mathrm{C}$ to $1080{ }^{\circ} \mathrm{C}$ (see Figure $1 \mathrm{a}$ ). At the same time, the AR, i.e. height divided by width, is enhanced by a factor of more than 8 . With respect to AR and dislocation annihilation, higher temperatures seem to be favorable. However, higher temperatures lead to a deterioration of the filling process and hence reduce the homogeneity: at $1000{ }^{\circ} \mathrm{C}$ all patterns are completely filled by fins without any interruptions along their length (see Figure $1 \mathrm{~b}$ ), whereas at $1100^{\circ} \mathrm{C}$ just a few patterns are partly filled by fins (see Figure $1 \mathrm{c}$ ). Due to this large inhomogeneity and the short fin lengths, a statistical evaluation of the fins grown at $1100{ }^{\circ} \mathrm{C}$ is not possible. In order to explain the influence of the temperature on the structural evolution of the fins, literature growth results from a-plane GaN layers on r-sapphire are analyzed in the following. On those aplane surfaces stripes elongated in c-direction could be observed[9],[10],[11]. These stripes can be also considered as surface undulations in the m-direction. The reason for these stripe features could be explained by density-functional theory calculations from Lymperakis and Neugebauer who found a large anisotropy in the diffusion barriers of $\mathrm{Ga}$ adatoms on a-plane $\mathrm{GaN}$ surfaces in the c-direction $(0.32 \mathrm{eV})$ in 
comparison to the $\mathrm{m}$-direction $(0.63 \mathrm{eV})[12]$. Thus, the diffusion in c-direction is faster than in m-direction, leading to the stripes extended in c-direction. Ni et al.[11] noted even more dominant undulations on aplane $\mathrm{GaN}$ layers with raising temperature and therefore suggested that the difference of the adatom migration lengths in $\mathrm{c}$ - and $\mathrm{m}$-direction is even increasing with temperature. Comparing these results with the growth of fins with a-plane sidewall surfaces, this model seems to be corroborated: low migration barriers of the Ga adatoms along c-direction would lead to a faster diffusion of growth species to the top facets and therefore to larger vertical growth rates. Higher migration barriers along m-direction would lead to interruptions along the fin length and therefore increase the inhomogeneity. Both becomes more and more apparent with raising growth temperatures, sustaining the theory of Ni et al.[11].

Another interesting result is the change of the top surface morphology as a function of temperature. After growth at a temperature of around $1020^{\circ} \mathrm{C}$ the fins have completely flat top surfaces after $1800 \mathrm{~s}$ of growth, whereas the top surface of fins grown at higher temperatures shows large roughness (see insets in Figure $1 \mathrm{a}$ ). It seems that the smaller growth rates at lower temperatures lead to a flattening of the top surface. A smooth surface is very important for the growth of quantum wells (QWs) for e.g. optoelectronic applications since rough surfaces can lead to high densities of voids, dislocations and stacking faults in the QW shell, as will be discussed later. Moreover, smooth surfaces are a necessary precondition for further device processing.

\subsection{Pressure}

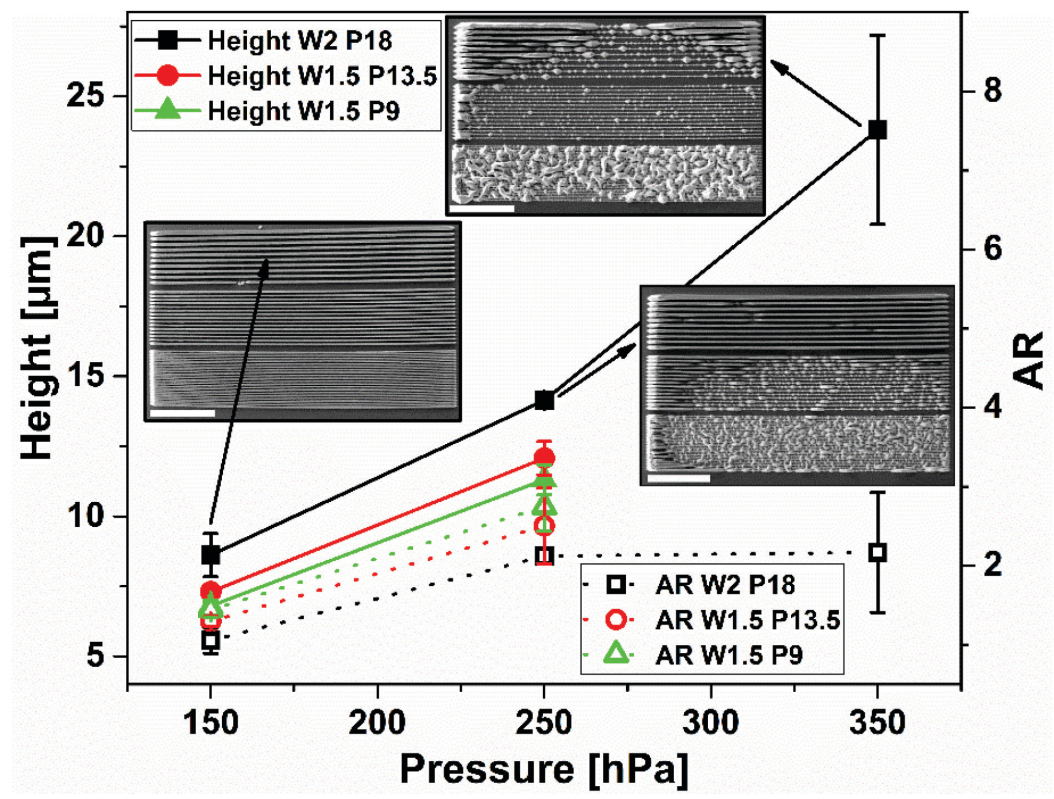

Figure 2: Fin height (solid symbols and lines) and AR (open symbols and dashed lines) over reactor pressure during 3D-growth-step. For a pressure of $350 \mathrm{hPa}$ just fins in pattern W2 P18 evolved. The insets show SE images of patterns W2 P6 (at the bottom in insets), W2 P12 and W2 P18 (at the top) with a scale bar length of $200 \mu \mathrm{m}$ each.

With increasing reactor pressure, the height of the fins is increasing (see Figure 2) whereas the homogeneity along the length of the fins as well as across different patterns is decreasing (see insets in Figure 2). This trend is similar to the one observed with increasing growth temperature. The width of the fins stays almost constant for low pressure values of 150 and $250 \mathrm{hPa}$, but it is increasing for a pressure of $350 \mathrm{hPa}$, with the consequence that the $\mathrm{AR}$ does not depend much on the reactor pressure in this range (see Figure 2), even though the height is increasing. A similar influence of the reactor pressure has been published for epitaxial lateral overgrowth (ELOG) experiments, although one needs to mention that the applied V/III ratio during ELOG is much higher than that for fin growth: By increasing the reactor pressure, the vertical growth rate of GaN stripes along m-direction is enhanced whereas the lateral growth rate, i.e. in non-polar as well as in semipolar directions, is reduced[13],[14].

\subsection{V/III Ratio}


For all V/III ratios used in the 3D-growth-step in these experiments - ranging from 30 to 130 - fin growth can be observed. This proves the wide parameter window for SAG of GaN fins. In order to analyze the overall efficiency of $\mathrm{Ga}$ and $\mathrm{N}$ incorporation, the total fin volume was determined and a corresponding layer thickness (CLT) was calculated, which gives the possibility to compare the results with planar growth rates of closed layers. The CLT is the thickness of a virtual planar thin film with the same total GaN volume as the $3 \mathrm{D}$ fin structures under investigation.
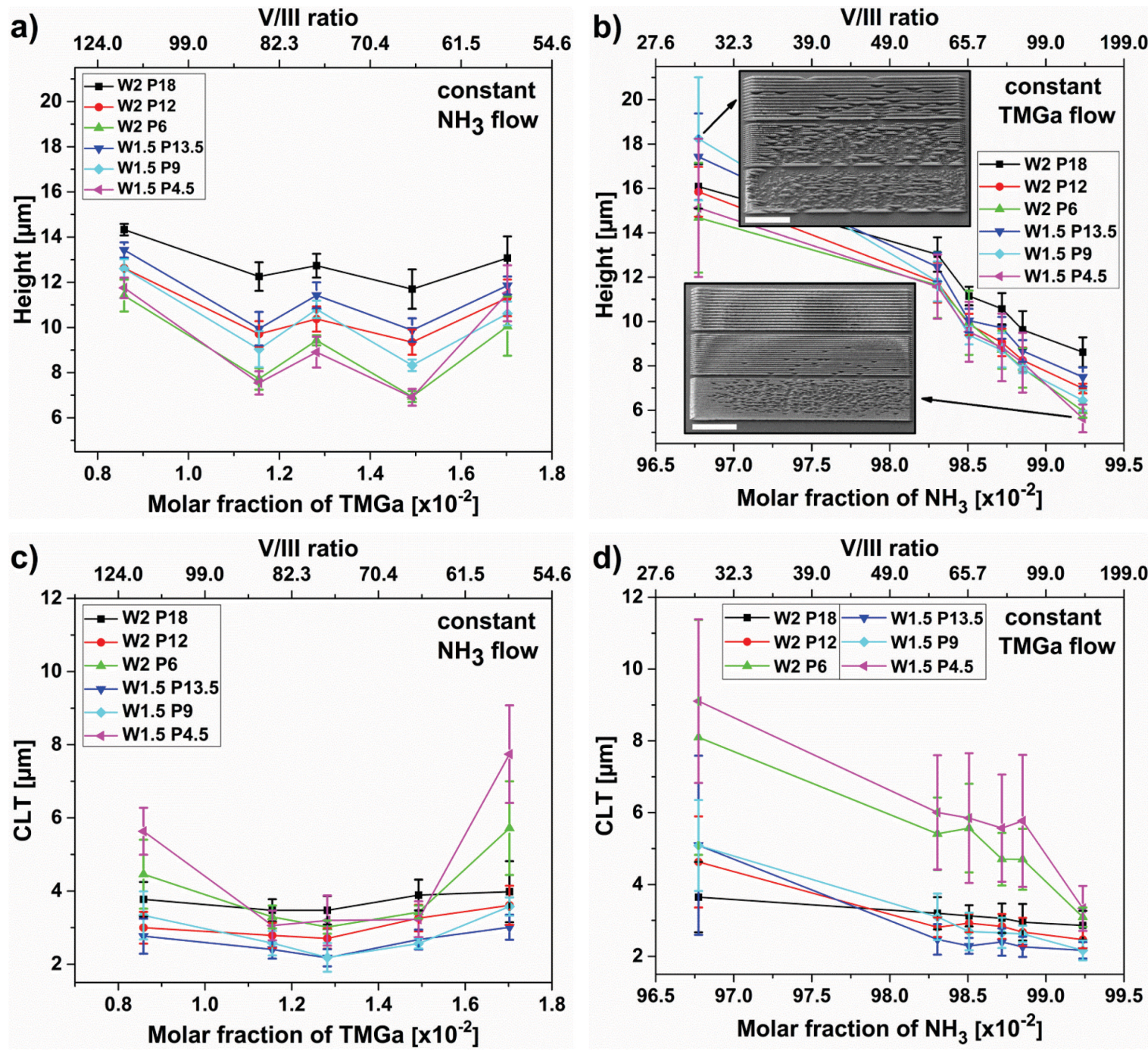

Figure 3: Fin height versus the $\mathrm{TMGa}$ (a) and $\mathrm{NH}_{3}$ (b) molar fraction of the total group-V and -III precursors. CLT of fins versus the TMGa (c) and $\mathrm{NH}_{3}$ (d) molar fraction. The insets in (b) display SE images of the patterns W1.5 P4.5 (at the bottom in insets), W1.5 P9 and W1.5 P13.5 (at the top) of the two samples grown at a V/III ratio of 30 and of 130 , respectively. The scale bar has a length of $200 \mu \mathrm{m}$ in both cases.

In a range of TMGa flows from 89 to $179 \mu \mathrm{mol} / \mathrm{min}$, no large influence of TMGa molar fraction on the fin height and width can be observed. The height of the fins showed random variations of less than $40 \%$ in total (see Figure 3 a). The CLT is almost constant, except for some deviations (Figure $3 \mathrm{c}$ ). For a diffusionlimited growth process, an increase in growth rate with increasing TMGa would be expected as it will be discussed later. Nevertheless, this effect does not seem to be unambiguously detectable for the relatively small variation of the TMGa flow experimented here.

When increasing the $\mathrm{NH}_{3}$ mole fraction while keeping the TMGa flow constant, the V/III ratio is increasing. As a consequence, a distinct trend becomes visible in Figure $3 \mathrm{~b}$ : The higher the amount of $\mathrm{NH}_{3}$ in the gas phase, the less vertical growth rate is observed. At the same time the lateral growth rate increases: The width of the fins is slightly increasing (not shown here), corresponding to an enhanced 
growth rate in a-direction, and the homogeneity along the fins is also increasing (see insets in Figure $3 \mathrm{~b}$ ), due to an enhanced growth rate in m-direction. A decreasing height and increasing width result in a significantly reduced AR. The decrease of vertical growth rates at relatively high V/III ratios or high $\mathrm{NH}_{3}$ flows has meanwhile been demonstrated by many groups working on $\mathrm{GaN}$ nanorods[15],[16] or ELOG[17],[18]. For higher $\mathrm{NH}_{3}$ molar fraction, the CLT is slightly decreasing (Figure $3 \mathrm{~d}$ ). A possible explanation is that a higher $\mathrm{NH}_{3}$ flow leads to higher active $\mathrm{H}$ concentration, produced by cracking of $\mathrm{NH}_{3}$. The increased amount of active $\mathrm{H}$ can cause a passivation of the surfaces, which could lead to enhanced desorption of species and therefore reduced growth rates[19]. On the other hand, active $\mathrm{H}$ could etch $\mathrm{GaN}$ especially at the high temperatures applied here[20]. Besides of the increased amount of active $\mathrm{H}$, higher $\mathrm{NH}_{3}$ flows could suppress the diffusion of In- and Ga-containing species in the gas phase. All these effects could lead to a reduction of the CLT with increased $\mathrm{NH}_{3}$ molar fraction.

In our case, for the SAG of fins, a V/III ratio of about 77 has been identified to be the best compromise between sufficient height and homogeneity.

\subsection{Total Precursor Flows}

In order to investigate the influence of the total precursor flows on the fin CLT while keeping the V/III ratio constant at 77, the flows of both $\mathrm{TMGa}$ and $\mathrm{NH}_{3}$ were increased. Thus, the $\mathrm{TMGa}$ flow was varied by almost a factor of 10 , from $67 \mu \mathrm{mol} / \mathrm{min}$ up to $446 \mu \mathrm{mol} / \mathrm{min}$, and the $\mathrm{NH}_{3}$ flow was varied accordingly. Within this sample series, two different $\mathrm{NH}_{3}$ MFCs had to be used in order to reach all intended flow values (see dashed and solid lines in Figure 4, respectively). For the lowest flow, no growth of fins could be observed and the stripe openings showed a rough surface (see cross-section in inset in Figure 4), most probably due to back etching of the underlying GaN buffer. In this case, the growth rate seems to be compensated by the etching rate, caused by high temperature and $\mathrm{H}_{2}$ carrier gas in combination with a low TMGa flow[21]. Increasing the TMGa and $\mathrm{NH}_{3}$ flows, fins start to evolve. In the low flow regime with TMGa flows below approximately $180 \mu \mathrm{mol} / \mathrm{min}$ (under constant V/III ratio of 77) the influence on the CLT is marginal, being consistent to the evaluation of the V/III ratio by changing the III-source flow (Figure $3 \mathrm{c}$ ). However, for higher flows, height and width simultaneously increase, leading to a rather constant AR, but the CLT increases distinctly (Figure 4): It almost doubles for an increase of TMGa flow from 134 to $446 \mu \mathrm{mol} / \mathrm{min}$. In ELOG experiments increased width and height with enhanced TMGa were shown[22] which is expected for a diffusion-limited growth process. Although the $\mathrm{NH}_{3}$ flow increased continuously along with the TMGa flow, the passivation, etching or diffusion-limiting effect of $\mathrm{NH}_{3}$, which was discussed in the previous chapter, seems to have a smaller effect compared to the growth rate increase due to more effective III-species (at least for TMGa flows larger than $180 \mu \mathrm{mol} / \mathrm{min}$ ). For TMGa flows above approximately $250 \mu \mathrm{mol} / \mathrm{min}$, the fins show small semipolar $\{11-22\}$ facets at the top of the sidewalls instead of purely non-polar $\{11-20\}$ planes (see cross-section in upper inset in Figure 4). A possible explanation could be that the growth rate of the $\{11-22\}$ facets is saturating earlier than the one of the other facets[17]. Therefore, with higher total growth rate, the $\{11-22\}$ facet will become more pronounced. For the SAG of GaN fins, total flows of $223 \mu \mathrm{mol} / \mathrm{min} \mathrm{TMGa}$ and $17.2 \mathrm{mmol} / \mathrm{min} \mathrm{NH}_{3}$ are chosen in order to achieve not too wide, but relatively high fins with purely non-polar sidewalls (see lower right inset in Figure 4). 


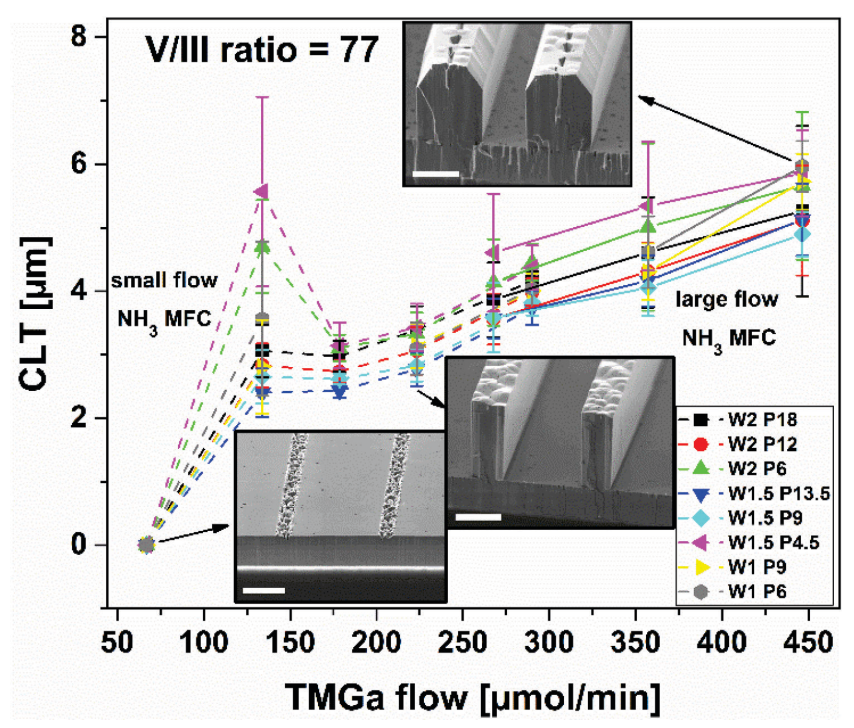

Figure 4: Corresponding layer thickness (i.e. fin volume per substrate area) over the TMGa flow at a constant $\mathrm{V} / \mathrm{III}$ ratio of 77 . Dashed (solid) lines connect the points for samples grown by employing the small (large) $\mathrm{NH}_{3}$ MFC. Insets show SE images of cross-sections in pattern W2 P12 with TMGa flows of 67,223 and $446 \mu \mathrm{mol} / \mathrm{min}$, respectively. The scale bar has a length of $5 \mu \mathrm{m}$ in all cases.

\subsection{2-temperature-step-growth}

In view of all these findings about fin growth, we developed a 2-temperature-step-growth method in order to grow high AR fins with large homogeneity as well as with smooth sidewall and top surfaces. After the filling-step, which is described in the section "Materials and Methods", two different growth modes for the 3D-growth-step were applied. In the first step, growth conditions for guaranteeing a good homogeneity over the patterns as well as over the fin length were used: a low temperature of $1020^{\circ} \mathrm{C}$ and a moderate pressure of $250 \mathrm{hPa}$. After $300 \mathrm{~s}$ of this first step, a fin with relatively smooth top surface evolves (see Figure $5 \mathrm{a}$ ). In the second step, the pressure was reduced to $150 \mathrm{hPa}$ in order to keep the good homogeneity from the first 3D-growth-step. Additionally, the temperature was increased to $1060{ }^{\circ} \mathrm{C}$ for higher crystal quality and to keep the growth rate large despite the reduced pressure. This second 3D-growth-step is applied for $2700 \mathrm{~s}$. Both temperature steps were performed under the increased precursor flows of 223 $\mu \mathrm{mol} / \mathrm{min} \mathrm{TMGa}$ and $17.2 \mathrm{mmol} / \mathrm{min} \mathrm{NH}_{3}$ for further enhancing the growth rate. The high AR, good homogeneity as well as smooth sidewalls and top surfaces emerging from this 2-temperature-step-growth can be seen in Figure $5 \mathrm{~b}$ and c. With vertical growth rates of more than $10 \mu \mathrm{m} / \mathrm{h}$ large heights of $32 \mu \mathrm{m}$ and ARs greater than 4 can be reached e.g. in pattern W2 P12 within three hours of growth.

On the fins with flat top surfaces, e.g. the 2-temperature-step-grown fins in Figure 5 b, local charging effects indicating reduced conductivity in some regions can be observed by SEM. Vertical holes inside the fins lead to less charging in their direct surroundings, which can be seen by the irregular contrast on the top facets in Figure $5 \mathrm{~b}$. Based on CL analysis we assume that these charging effects are due to different regions within the fins which have different incorporation rates of impurities or dopants. This will be discussed in detail in another publication, which will focus on the electro-optical properties of fin structures.

In order to analyze the optical quality of the fins, CL measurements were performed. Electron beam excitation of the GaN buffer layer can be avoided by scanning over a region at the upper part of the fins. Besides of a broad yellow defect emission band, a distinct $\mathrm{GaN}$ near band edge emission peak can be detected at the a-plane sidewalls of the fins (see Figure $5 \mathrm{~d}$ ). 

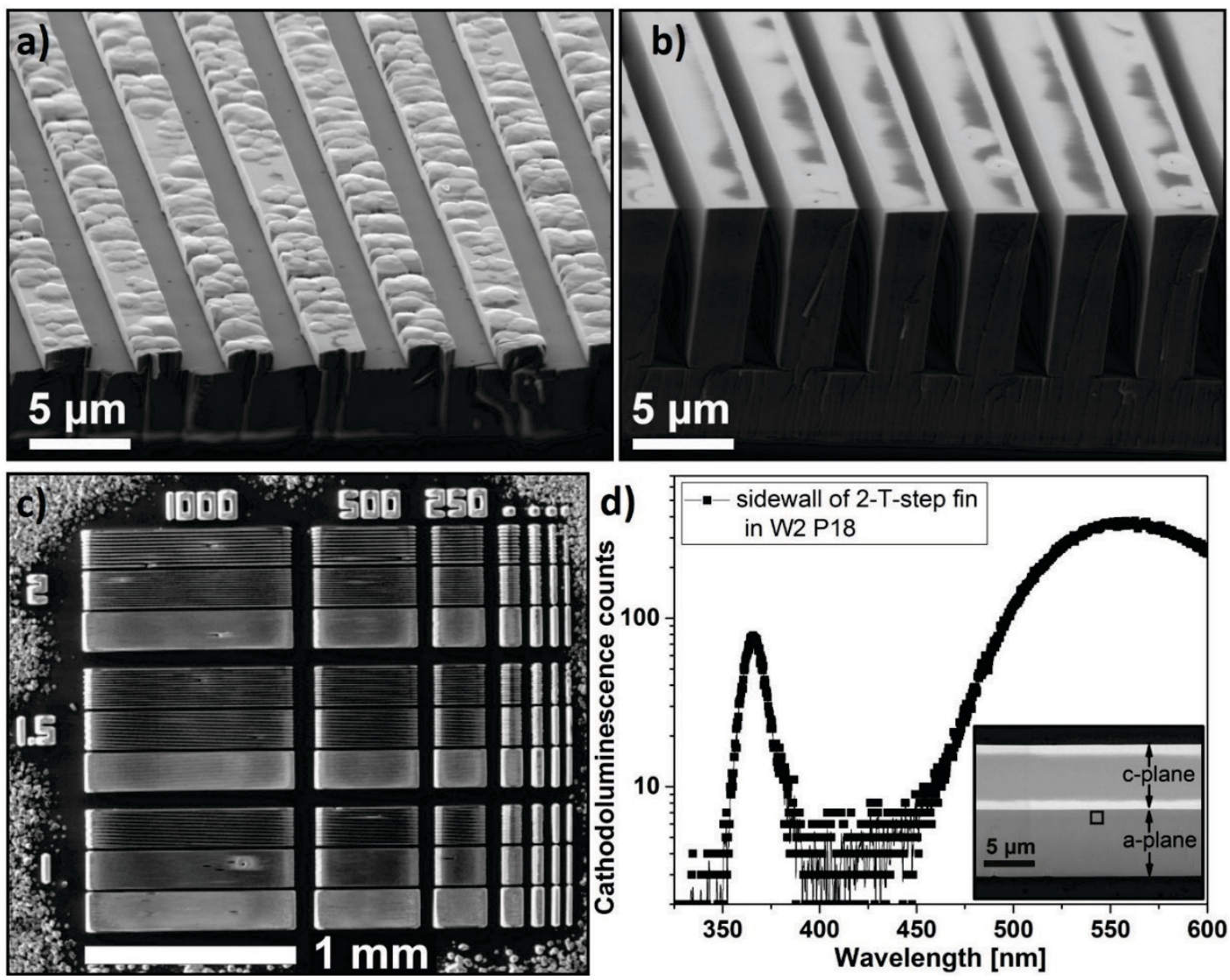

Figure 5: Cross-section SE images of fins grown with the first 3D-growth step only (a) and grown with the complete 2-temperature-step-growth (b) in pattern W1.5 P4.5 at a tilt of $60^{\circ}$, overview SE image of 2temperature-step-growth (c), CL spectrum (d) measured from a scanning area at the upper sidewall of a fin in pattern W2 P18 (inset, SE side-view image of the fin with a tilt of $30^{\circ}$ )

\subsection{Fin LED}

In order to fabricate core-shell fin LED structures, two different kinds of fin cores were used: On the one hand, the GaN fin cores were grown with the standard 3D-growth-step conditions, as mentioned in the section "Materials and Methods". These cores, which show a high roughness of the top facet, were overgrown by a shell with a single InGaN QW and p-GaN capping layers (more details in section "Materials and Methods"). The structures are marked in the following as "fin LED A". On the other hand, fin cores were grown by the new 2-temperature-step-growth in order to improve the surface smoothness. The 2-temperature-step grown fins were covered by a shell under very similar growth conditions as fin LED A and are called "fin LED B" in the following. 

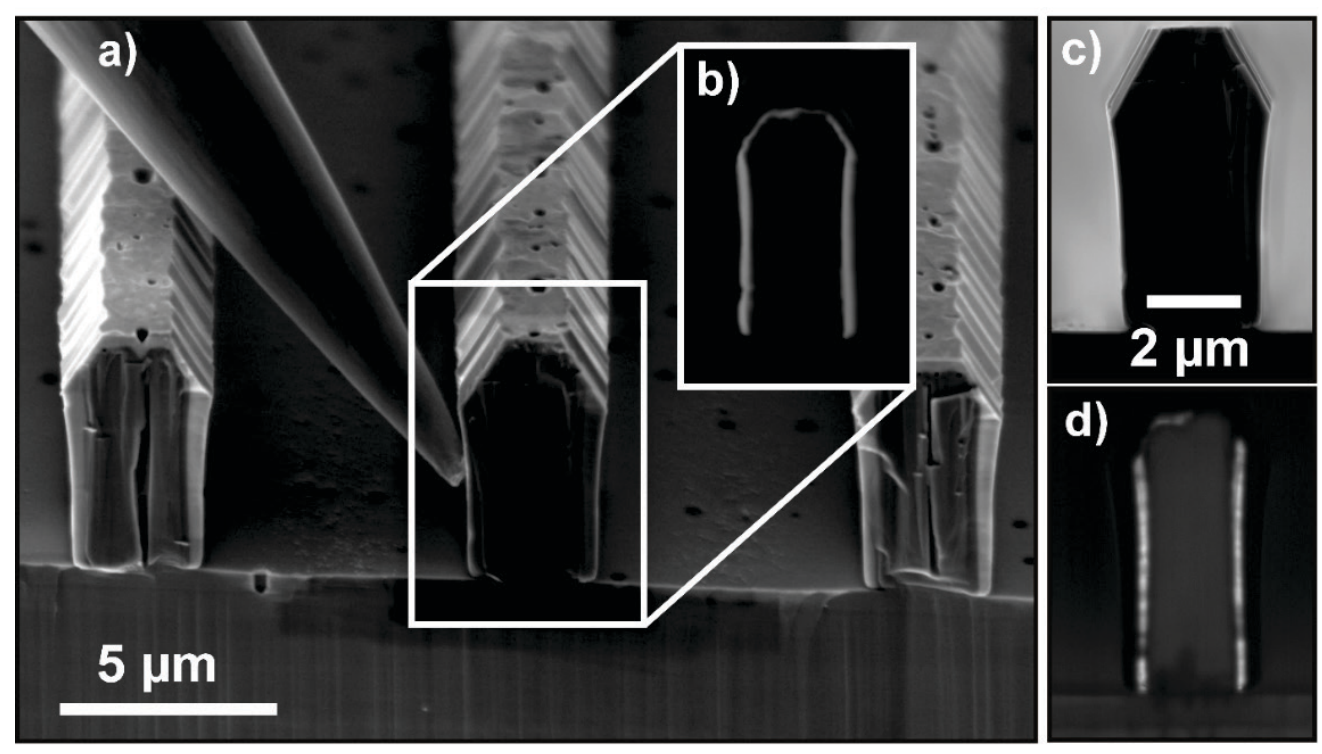

Figure 6: SE images of fin LEDs A in pattern W1.5 P9 with a tilt of $60^{\circ}$ (a) and $90^{\circ}$ (c), corresponding EBIC at a reverse voltage of $2 \mathrm{~V}$ (inset b) and panchromatic CL (d), respectively.

The shell surface of fin LED A shows high roughness (Figure 6 a), most probably originating from the high roughness of the fin cores' top facets. Nevertheless, the p-type shell is entirely wrapped around the n-doped fin core, as can be shown by EBIC measurements. For these, the p-side of a single fin LED structure was brought in contact to a tungsten probe tip (Figure 6 a) while the n-GaN buffer layer was connected via the sample holder, and a reserve bias of $2 \mathrm{~V}$ was applied across the pn-diode. The EBIC signal (Figure $6 \mathrm{~b}$ ) shows a bright stripe related to the depletion zone of the pn-junction, which obviously is wrapped around the whole fin structure. Therefore, the $\mathrm{p}-\mathrm{GaN}$ shell conformably covers the sidewalls as well as the top facets. CL measurements of the fin LED A prove that the InGaN QW is also covering the complete sidewalls of the fins (see panchromatic CL in Figure $6 \mathrm{~d}$ ) which is difficult to realize: dense ensembles of all 3D architectures like columns or fins with high ARs are leading to a possible depletion of precursors at the bottom of the structures during their shell growth. The area from where material can diffuse to the fins is even more reduced compared to core-shell column LEDs: the gas volume as well as the mask surface per fin surface is smaller than that for columns. The QW emission for the fin LED A shows a maximum at a wavelength of around $420 \mathrm{~nm}$. For GaN core-shell column LEDs it was reported that due to a gradient in the InGaN composition the emission from the sidewall QW is red shifted from the bottom to the top[23],[24],[25],[26],[27]. The same trend is also observed for fin LEDs (not shown here).

For more detailed analysis, TEM was performed on fin LED A (Figure 7). Selected area electron diffraction (SAD) patterns prove the GaN hexagonal crystal structure where the longitudinal direction of the fin is aligned along the crystallographic $<-1100>$-direction (see inset of Figure 7 a). Based on the bright-field STEM image in Figure 7 a, the dislocation density in the fin core can be estimated to approx. 2 $\times 10^{8} \mathrm{~cm}^{-2}$ assuming a TEM lamella thickness of $0.2 \mu \mathrm{m}$. This value for the dislocation density is remarkably low. Furthermore, the threading dislocation density through the active region along the a-plane sidewall is even lower since there are just very few dislocations bending to the sidewalls (Figure $7 \mathrm{a}$ ).

However, TEM shows that there are lots of dislocations and nanovoids in the top c-plane shell (Figure $7 \mathrm{~b}$ ). In the region of the a-plane sidewalls close to the top there is a high density of stacking faults (SFs) emerging at the interface between core and shell (Figure $7 \mathrm{c}$ ). The formation of such SFs at the QW interface was already reported for GaN/InGaN 3D column LEDs[28],[29]. These stacking faults have a negative effect on the sidewall surface morphology since they lead to steps and surface roughening of the aplane sidewalls of the fin LED A. 


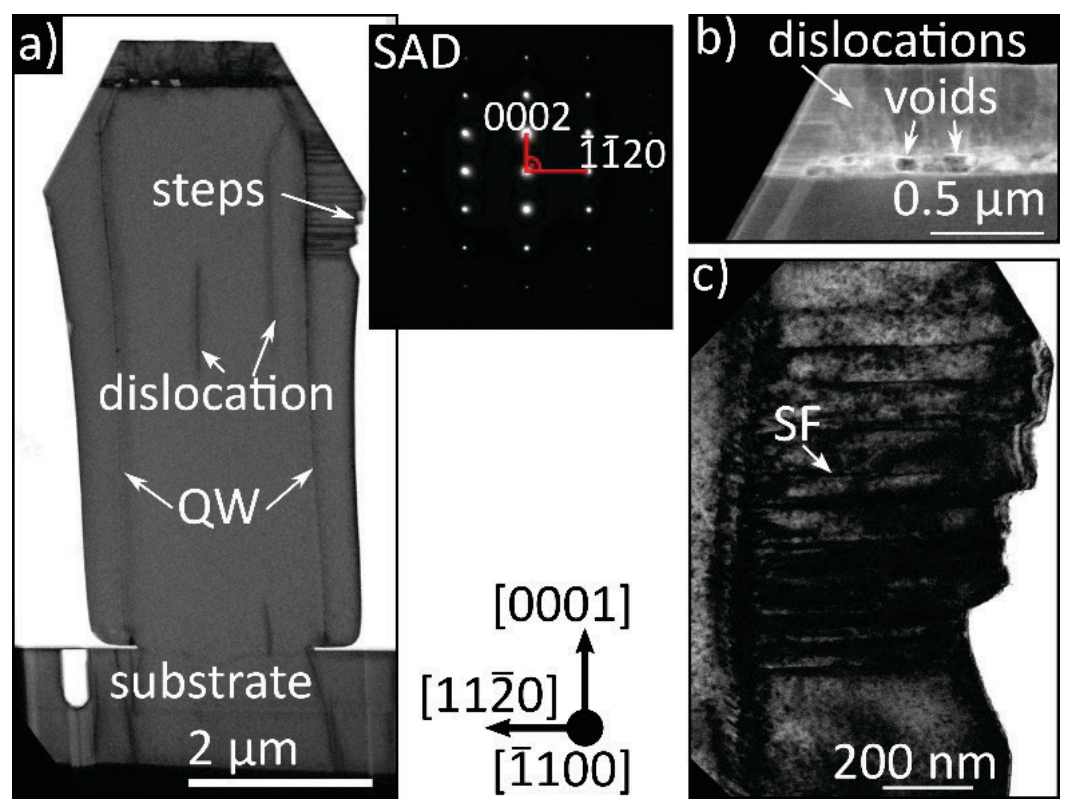

Figure 7: (a) Bright-field STEM micrograph revealing the microstructure of fin LED A. The inset shows an SAD pattern along $<\overline{1} 100>$ zone axis of the core region proving the hexagonal crystal symmetry around the vertical axis. (b) HAADF STEM micrograph of the top region of the fin. (c) Magnified TEM image reflecting SFs and surface steps. The depicted crystallographic orientation is the valid for all micrographs.

In order to circumvent the high surface roughness of the fin LEDs, a second LED based on the 2temperature-step-growth of the core, was grown (fin LED B). As expected, SEM images show much smoother sidewalls as well as top facets for the fin LED B (inset in Figure 8). EBIC of the fin LED B again proves a conformal p-GaN shell (not shown here). However, the QW is not efficiently present on the whole sidewall surface but just on the upper part of it. This is because the distribution of the InGaN QW is depending on the fin height (larger height leads to less InGaN coverage) and the pitch (larger pitch leads to more InGaN coverage). The width and height of fin LED B are much larger compared to the one of fin LED A. Thus, less sidewall area of fin LED B is covered with a QW, if the same pattern is investigated. The influence of different patterns was clearly found by panchromatic CL images: Although the fin cores are typically higher for larger pitches (due to larger gas phase volume and mask surface area), more absolute as well as relative area of the sidewalls is covered with increasing pitch: For a pitch of $18 \mu \mathrm{m}$ in pattern W2 P18, which is corresponding to a distance between the final fin LEDs of $9.7 \mu \mathrm{m}$, the complete sidewall can be covered. If the pitch is reduced to $12 \mu \mathrm{m}$, corresponding to $5.5 \mu \mathrm{m}$ distance, roughly the upper half of the sidewall is covered. For a pitch of $6 \mu \mathrm{m}$, corresponding to a distance of only $1.3 \mu \mathrm{m}$, just the upper sixth fraction of the total height seems to be covered. These estimations need to be confirmed by further TEM analysis.

After contacting the a-plane sidewall of a single fin LED B with the tungsten probe tip (inset in Figure 8), a current in forward direction was applied to the LED structure while the electron beam is blanked in order to avoid simultaneous CL. Using the micromanipulator setup in the CL setup, the electroluminescence (EL) was measured. The EL spectra for increasing current from $2 \mu \mathrm{A}$ to $22 \mu \mathrm{A}$ are presented in Figure 8, their intensities are monotonously increasing versus driving current. Due to the relatively low conductivity of the thin $\mathrm{p}-\mathrm{GaN}$ shell layer, lateral current components will cause a significant voltage drop accompanied by current crowding close to the probe tip. Two peaks related to InGaN emission are visible in the EL spectra, indicated in Figure 8. The higher-wavelength peak around $535 \mathrm{~nm}$ is interpreted to originate from the junction at the semi-polar facet at the fin top (or at the transition from non-polar to semi-polar facet) which usually contains a QW (or an InGaN wire) with a significantly higher In-content than on the non-polar sidewall facet[27]. This enhanced Indium content could be e.g. caused by different strain states at the transition between facets[30],[31]. The junction in this position would have a lower onset potential which leads to a dominant EL emission around $535 \mathrm{~nm}$ at low injection currents[32]; in particular using a point contact placed on the top part of the sidewall. With increasing driving current, also the voltage drop along the $\mathrm{p}-\mathrm{GaN}$ shell increases and the sidewall junction with a higher onset potential will be driven, too, 
emitting EL around $410 \mathrm{~nm}$. With further increase of the injection current, this junction at the non-polar facet close to the probe tip will carry most of the additional current - resulting in a saturation of the EL emission from the semi-polar facet. Based on EL (local current injection) and CL (local excitation by ebeam) at different positions it was found, that the peak wavelength of the non-polar sidewall QW is red shifting along the height of the fins, as discussed earlier. Besides the two InGaN peaks, also in EL a broad yellow luminescence (YL) band is visible at higher wavelengths. Its intensity saturation versus the driving current is assigned to a saturation of the defect-related recombination channel.

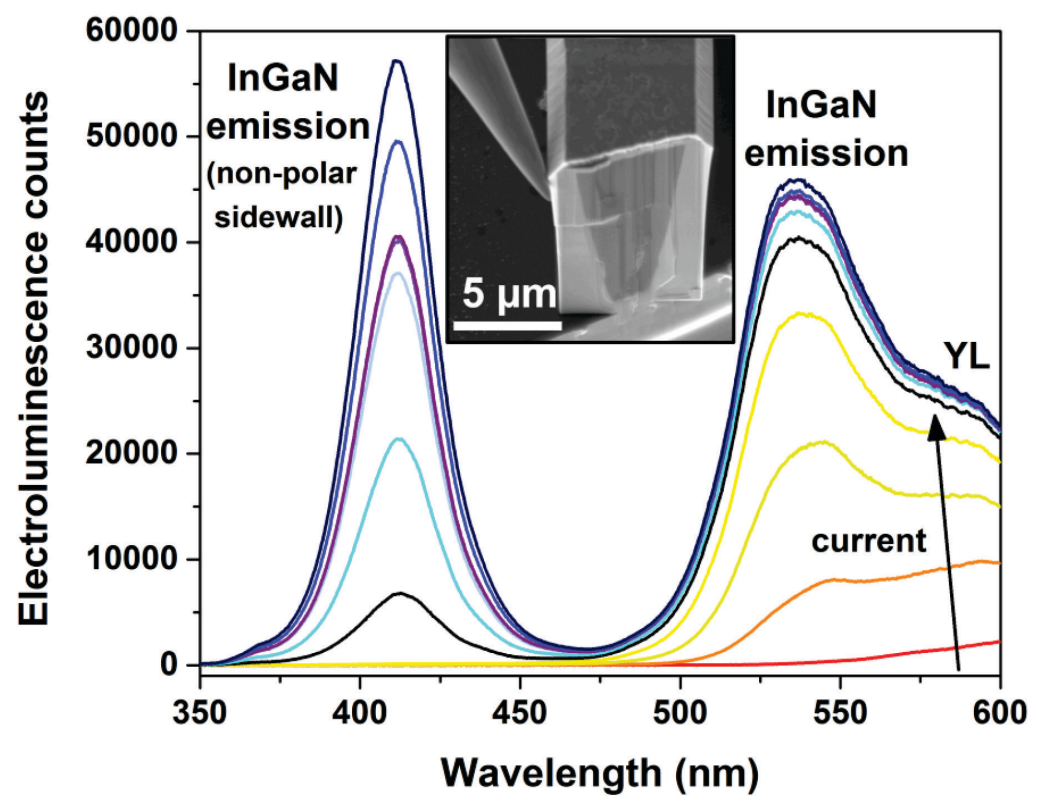

Figure 8: Electroluminescence spectra taken by the CL setup. A tungsten probe tip is contacting the upper part of the non-polar sidewall of fin LED B (see SE image under $30^{\circ}$ tilt in the inset) and a forward current increasing from $2 \mu \mathrm{A}$ to $22 \mu \mathrm{A}$ (in $2 \mu \mathrm{A}$ steps) was applied, subsequently.

TEM analysis of fin LED B has detected a larger number of dislocations in the fin core compared to fin LED A (Figure 9 a). This can probably be explained by the increased sample thickness and by the larger width of the fin. However, just one of these dislocations is bending to the sidewall and could degrade the QW efficiency in this area. The shell on the c-plane top facet still shows an increased defect density, but the amount of nanovoids seems to be reduced compared to fin LED A (Figure $9 \mathrm{~b}$ ). In the sidewall shell, no SFs were found at all (Figure $9 \mathrm{c}$ ). This could be attributed to the improved interface quality due to the 2temperature-step-growth of fins. 

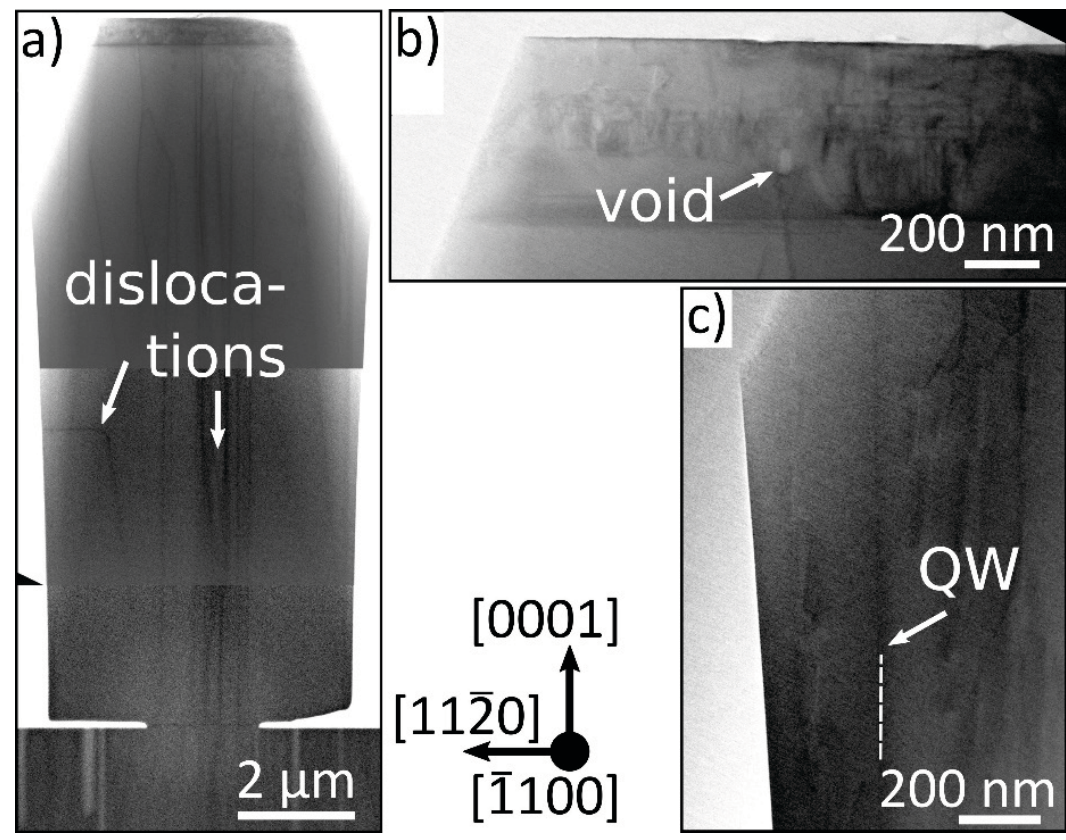

Figure 9: Bright-field STEM cross-sectional micrographs of a fin LED B based on a 2-temperature-stepgrowth of the fin core. (a) Micrograph of the entire height of a fin revealing the propagation of dislocations within the fin. Due to the large dimension and thickness gradients of the sample three single micrographs were merged together and the contrast was improved. (b) Micrograph of the c-plane top facet revealing a lower density of nanovoids and a more homogeneous core-shell interface compared to fin LED A. (c) Micrograph of the left sidewall showing the absence of SFs and surface steps. The depicted crystallographic orientation is the valid for all micrographs.

Recapitulating, the growth of fin LEDs was demonstrated, but it is critical for rough core surfaces and large fin heights. While the surface smoothness of the core can be improved e.g. by the suggested 2-temperaturestep-growth, the height is mandatory to achieve high ARs. Large heights of 3D architectures lead to incomplete InGaN coverage of their sidewalls. This challenge seems to be more critical for fins than for columns: Besides of the reduced gas phase and mask surface for fins (as discussed before), the top facets of these two kinds of 3D architectures are quite diverse: On columns, non-polar $\{10-10\}$ and semi-polar $\{10-$ 11 facets are available, with the growth rate being slower on the semi-polar facets than on the non-polar facets[33]. In contrast, fin cores are terminated by non-polar $\{11-20\}$ and polar $\{0001\}$ facets. Here, the growth rate on the polar facet is much higher than on the non-polar one, which was observed also by TEM of fin LEDs. Additionally, the Indium incorporation efficiency is almost doubled on c-planes in comparison to a-planes[34]. Both effects lead to much stronger trapping of In-containing species at the top facets of fins. Thereby, less material can diffuse to the non-polar sidewalls and the InGaN layers are thinner on fins. As a consequence, the distribution along the height is worse for fins. In order to achieve more homogenous luminescence along the complete sidewalls, an optimization of QW growth on a-plane fins is ongoing at the moment. First results show, that the total amount of Indium on the sidewalls can be increased by lowering the temperature or increasing the trimethylindium flow. However, the gradient along the height is not reduced by these methods. Hence, very high Indium concentrations at the top of the non-polar sidewalls occur and lead to reduced efficiencies in these regions. More detailed results about the QW optimization of fin LEDs will be published elsewhere.

\section{Conclusions}

The influence of continuous-flow selective area MOVPE parameters on the growth of GaN fins with largescale non-polar $\{11-20\}$ sidewalls was analyzed. With increasing temperature or increasing pressure the vertical growth rate of the fins is increasing, but the homogeneity along their length as well as over the different mask patterns is decreasing. As a possible reason for this temperature dependence, the differences in diffusion barriers for $\mathrm{Ga}$ adatoms on a-plane surfaces in $\mathrm{c}$ - and $\mathrm{m}$-directions have been discussed.

Smooth top surfaces of fins can only be achieved at lower temperatures. The V/III ratio can be varied in a relatively large range from approximately 60 to 120 without deteriorating the fin morphology considerably. 
While keeping the V/III ratio constant at 77, an increase of the precursor flows of both $\mathrm{TMGa}$ and $\mathrm{NH}_{3}$ can distinctly enhance the growth rate as well as the corresponding layer thickness of the fins. In order to achieve homogenous fins with smooth sidewalls as well as top facets, a 2-temperature-step-growth was developed. Core-shell growth of GaN/InGaN core-shell LED heterostructures was realized. The fin LEDs were characterized by EBIC, CL, EL and TEM and show a conformal p-GaN shell. The dislocation density in the fin core is estimated to be approx. $2 \times 10^{8} \mathrm{~cm}^{-2}$ and the threading dislocation number on the a-plane sidewalls is even much lower. The surface of the fin LED is much smoother for the 2-temperature-stepgrowth of fin cores. The EL spectra with InGaN and YL emission peaks and their current dependence have been explained by local onset potentials of the junction and current crowding.

\section{Acknowledgment}

We would like to acknowledge the financial support of the DFG within the research unit FOR1616 (Dynamics and Interactions of Semiconductor Nanowires for Optoelectronics) and the epitaxy competence center $\mathrm{ec}^{2}$. We thank the EMRP, which is jointly funded by the EMRP participating countries within EURAMET and the European Union, for financial support within the ENG62 MESaIL project.

Additionally, we acknowledge financial support from "Niedersächsisches Vorab" through "Quantum- and Nano-Metrology (QUANOMET)" initiative within the project NL2. The scholarship support by the China Scholarship Council is highly acknowledged. Moreover, we thank Juliane Breitfelder, Karl-Heinz Lachmund, Manfred Karsten and Andreas Heidemann for technical support and Astrid Pfeiffer for the TEM lamella preparation.

[1] T. Akasaka, Y. Kobayashi, S. Ando, N. Kobayashi, GaN hexagonal microprisms with smooth vertical facets fabricated by selective metalorganic vapor phase epitaxy, Appl. Phys. Lett. 71 (1997) 2196. doi:10.1063/1.119379.

[2] T.-W. Yeh, Y.-T. Lin, B. Ahn, L.S. Stewart, P. Daniel Dapkus, S.R. Nutt, Vertical nonpolar growth templates for light emitting diodes formed with GaN nanosheets, Appl. Phys. Lett. 100 (2012) 33119. doi:10.1063/1.3671182.

[3] A.K. Rishinaramangalam, S.M. U1 Masabih, M.N. Fairchild, J.B. Wright, D.M. Shima, G. Balakrishnan, I. Brener, S.R.J. Brueck, D.F. Feezell, Controlled Growth of Ordered III-Nitride Core-Shell Nanostructure Arrays for Visible Optoelectronic Devices, J. Electron. Mater. 44 (2015) 1255-1262. doi:10.1007/s11664-014-3456-z.

[4] H.M. Ng, W. Parz, N.G. Weimann, A. Chowdhury, Patterning GaN microstructures by polarity-selective chemical etching, Japanese J. Appl. Physics, Part 2 Lett. 42 (2003). doi:10.1143/JJAP.42.L1405.

[5] S. Keller, C. Schaake, N.A. Fichtenbaum, C.J. Neufeld, Y. Wu, K. McGroddy, A. David, S.P. DenBaars, C. Weisbuch, J.S. Speck, U.K. Mishra, Optical and structural properties of GaN nanopillar and nanostripe arrays with embedded InGaN/GaN multi-quantum wells, J. Appl. Phys. 100 (2006) 54314. doi:10.1063/1.2234812.

[6] K. Kishino, A. Kikuchi, Group-III nitride structure and method for producing a group-III nitride structure, 2010.

[7] J. Hartmann, F. Steib, H. Zhou, J. Ledig, S. Fündling, F. Albrecht, T. Schimpke, A. Avramescu, T. Varghese, H.-H. Wehmann, M. Straßburg, H.-J. Lugauer, A. Waag, High Aspect Ratio GaN Fin Microstructures with Nonpolar Sidewalls by Continuous Mode Metalorganic Vapor Phase Epitaxy, Cryst. Growth Des. 16 (2016) 1458-1462. doi:10.1021/acs.cgd.5b01598.

[8] M. Niehle, A. Trampert, Electron tomography on nanopores embedded in epitaxial GaSb thin films, Micron. 73 (2015) 54-62.

[9] M.D. Craven, S.H. Lim, F. Wu, J.S. Speck, S.P. Denbaars, Structural characterization of nonpolar (1120) a-plane GaN thin films grown on (1102) r-plane sapphire, Appl. Phys. Lett. 81 (2002) 469-471. doi:10.1063/1.1493220.

[10] H. Wang, C. Chen, Z. Gong, J. Zhang, M. Gaevski, M. Su, J. Yang, M.A. Khan, Anisotropic structural characteristics of (11-20) GaN templates and coalesced epitaxial lateral overgrown films deposited on 
(10-12) sapphire, Appl. Phys. Lett. 84 (2004) 499-501. doi:10.1063/1.1644054.

[11] X. Ni, Y. Fu, Y.T. Moon, N. Biyikli, H. Morkoç, Optimization of (11-20) a-plane GaN growth by MOCVD on (1-102) r-plane sapphire, J. Cryst. Growth. 290 (2006) 166-170. doi:10.1016/j.jcrysgro.2006.01.008.

[12] L. Lymperakis, J. Neugebauer, Large anisotropic adatom kinetics on nonpolar GaN surfaces: Consequences for surface morphologies and nanowire growth, Phys. Rev. B - Condens. Matter Mater. Phys. 79 (2009) 1-4. doi:10.1103/PhysRevB.79.241308.

[13] H. Miyake, A. Motogaito, K. Hiramatsu, Effects of Reactor Pressure on Epitaxial Lateral Overgrowth of GaN via Low-Pressure Metalorganic Vapor Phase Epitaxy, Jpn. J. Appl. Phys. 38 (1999) L1000-L1002. doi:10.1143/JJAP.38.L1000.

[14] K. Hiramatsu, K. Nishiyama, M. Onishi, H. Mizutani, M. Narukawa, A. Motogaito, H. Miyake, Y. Iyechika, T. Maeda, Fabrication and characterization of low defect density GaN using facet-controlled epitaxial lateral overgrowth (FACELO), J. Cryst. Growth. 221 (2000) 316-326. doi:10.1016/S00220248(00)00707-7.

[15] P. Deb, H. Kim, V. Rawat, M. Oliver, S. Kim, M. Marshall, E. Stach, T. Sands, Faceted and Vertically Aligned GaN Nanorod Arrays Fabricated without Catalysts or Lithography, Nano Lett. 5 (2005) 18471851. doi:10.1021/nl0510762.

[16] J. Su, G. Cui, M. Gherasimova, H. Tsukamoto, J. Han, D. Ciuparu, S. Lim, L. Pfefferle, Y. He, A. V Nurmikko, C. Broadbridge, A. Lehman, Catalytic growth of group III-nitride nanowires and nanostructures by metalorganic chemical vapor deposition, Appl. Phys. Lett. 86 (2005) 13105. doi:10.1063/1.1843281.

[17] Frank Stefan Habel, Methoden zur Defektreduktion von Galliumnitrid-substraten und - Quasisubstraten, Cuvillier Verlag Göttingen, 2006.

[18] R. Zhang, L. Zhang, D.M. Hansen, M.P. Boleslawski, K.L. Chen, D.Q. Lu, B. Shen, Y.D. Zheng, T.F. Kuech, Epitaxial Lateral Overgrowth of GaN with Chloride-Based Growth Chemistries in Both Hydride and Metalorganic Vapor Phase Epitaxy, MRS Proc. 537 (1998) G4.7. doi:10.1557/PROC-537-G4.7.

[19] S.F. Li, S. Fuendling, X. Wang, S. Merzsch, M. a. M. Al-Suleiman, J.D. Wei, H.-H. Wehmann, a. Waag, W. Bergbauer, M. Strassburg, Polarity and Its Influence on Growth Mechanism during MOVPE Growth of GaN Sub-micrometer Rods, Cryst. Growth Des. 11 (2011) 1573-1577. doi: $10.1021 / \operatorname{cg} 101537 \mathrm{~m}$.

[20] K. Hiramatsu, H. Matsushima, H. Hanai, N. Sawaki, Selective Area Etching of GaN and AlGaN by Thermally Chemical Reaction in Hydrogen Ambient, MRS Proc. 482 (1997) 991. doi:10.1557/PROC482-991.

[21] E. V. Yakovlev, R.A. Talalaev, A.S. Segal, A. V. Lobanova, W. V. Lundin, E.E. Zavarin, M.A. Sinitsyn, A.F. Tsatsulnikov, A.E. Nikolaev, Hydrogen effects in III-nitride MOVPE, J. Cryst. Growth. 310 (2008) 4862-4866. doi:10.1016/j.jcrysgro.2008.07.099.

[22] O.H. Nam, T.S. Zheleva, M.D. Bremser, D.B. Thomson, R.F. Davis, Organometallic Vapor Phase Lateral Epitaxy of Low Defect Density GaN Layers, MRS Proc. 482 (1997) 301. doi:10.1557/PROC482-301.

[23] C.-H. Liao, W.-M. Chang, H.-S. Chen, C.-Y. Chen, Y.-F. Yao, H.-T. Chen, C.-Y. Su, S.-Y. Ting, Y.-W. Kiang, C.C. Yang, Geometry and composition comparisons between c-plane disc-like and m-plane coreshell InGaN/GaN quantum wells in a nitride nanorod, Opt. Express. 20 (2012) 15859-71. http://www.ncbi.nlm.nih.gov/pubmed/22772276.

[24] Xue Wang, Controlled growth of GaN columns and 3D core-shell LEDs by MOVPE, Technische Universität Braunschweig, 2014.

[25] M. Mandl, X. Wang, T. Schimpke, C. Kölper, M. Binder, J. Ledig, A. Waag, X. Kong, A. Trampert, F. Bertram, J. Christen, F. Barbagini, E. Calleja, M. Strassburg, Group III nitride core-shell nano- and microrods for optoelectronic applications, Phys. Status Solidi - Rapid Res. Lett. 7 (2013) 800-814. doi:10.1002/pssr.201307250.

[26] T. Krause, M. Hanke, Z. Cheng, M. Niehle, A. Trampert, M. Rosenthal, M. Burghammer, J. Ledig, J. 
Hartmann, H. Zhou, H.-H. Wehmann, A. Waag, Nanofocus x-ray diffraction and cathodoluminescence investigations into individual core-shell (In,Ga)N/GaN rod light-emitting diodes, Nanotechnology. 27 (2016) 325707. doi:10.1088/0957-4484/27/32/325707.

[27] T. Schimpke, H.-J. Lugauer, A. Avramescu, T. Varghese, A. Koller, J. Hartmann, J. Ledig, A. Waag, M. Strassburg, Position-controlled MOVPE growth and electro-optical characterization of core-shell InGaN/GaN microrod LEDs, in: H. Jeon, L.-W. Tu, M.R. Krames, M. Strassburg (Eds.), Proc. SPIE 9768, 2016: p. 97680T. doi:10.1117/12.2214122.

[28] R. Koester, J.S. Hwang, D. Salomon, X. Chen, C. Bougerol, J.P. Barnes, D.L.S. Dang, L. Rigutti, A. De Luna Bugallo, G. Jacopin, M. Tchernycheva, C. Durand, J. Eymery, M-plane core-shell InGaN/GaN multiple-quantum-wells on GaN wires for electroluminescent devices, Nano Lett. 11 (2011) 4839-4845. doi:10.1021/nl202686n.

[29] I. Griffiths, D. Cherns, X. Wang, H.H. Wehman, M. Mandl, M. Strassburg, A. Waag, Characterisation of 3D-GaN/InGaN core-shell nanostructures by transmission electron microscopy, Phys. Status Solidi Curr. Top. Solid State Phys. 11 (2014) 425-427. doi:10.1002/pssc.201300522.

[30] E.D. Le Boulbar, P.R. Edwards, S.H. Vajargah, I. Griffiths, I. G??rgel, P.M. Coulon, D. Cherns, R.W. Martin, C.J. Humphreys, C.R. Bowen, D.W.E. Allsopp, P.A. Shields, Structural and Optical Emission Uniformity of m-Plane InGaN Single Quantum Wells in Core-Shell Nanorods, Cryst. Growth Des. 16 (2016) 1907-1916. doi:10.1021/acs.cgd.5b01438.

[31] P.F. Lu, C. Sun, H.W. Cao, H. Ye, X.X. Zhong, Z.Y. Yu, L.H. Han, S.M. Wang, Strain induced composition profile in InGaN/GaN core-shell nanowires, Solid State Commun. 178 (2014) 1-6. doi:10.1016/j.ssc.2013.10.011.

[32] H. Zhang, G. Jacopin, V. Neplokh, L. Largeau, F.H. Julien, O. Kryliouk, M. Tchernycheva, Color control of nanowire InGaN/GaN light emitting diodes by post-growth treatment, Nanotechnology. 26 (2015) 465203. doi:10.1088/0957-4484/26/46/465203.

[33] T.W. Yeh, Y.T. Lin, L.S. Stewart, P.D. Dapkus, R. Sarkissian, J.D. O’Brien, B. Ahn, S.R. Nutt, InGaN/GaN multiple quantum wells grown on nonpolar facets of vertical GaN nanorod arrays, Nano Lett. 12 (2012) 3257-3262. doi:10.1021/nl301307a.

[34] K. Bang, S. Jung, K.H. Baik, J.-M. Myoung, Growth and characterization of single InGaN quantum well in nonpolar a-plane (11-20) InGaN/GaN light-emitting diodes, Curr. Appl. Phys. (2017). doi:10.1016/j.cap.2017.03.016. 